\title{
Medium-Term Outcomes of Laparoscopic Pubocervical Fascia Reconstruction and Sacrospinous Ligament Fixation for Pelvic Organ Prolapse
}

\section{Yunshan Zhu}

Zhejiang University, Hangzhou, China

\section{Xiao Zhang}

Zhejiang University, Hangzhou, China

\section{Danxia Chen}

Zhejiang University, Hangzhou, China

\section{Guangxiao Li}

Zhejiang University, Hangzhou, China

\section{Shanliang Shang}

Zhejiang University, Hangzhou, China

Jianqiong Li

Zhejiang University, Hangzhou, China

Jianhua Yang ( $\nabla$ yjh2006@zju.edu.cn )

Zhejiang University, Hangzhou, China https://orcid.org/0000-0002-7834-4001

Research article

Keywords: Pelvic organ prolapse, Laparoscopy, Pubocervical fascia reconstruction, Sacrospinous ligament fixation

Posted Date: October 9th, 2020

DOI: https://doi.org/10.21203/rs.3.rs-77031/v1

License: (c) (1) This work is licensed under a Creative Commons Attribution 4.0 International License. Read Full License 


\section{Abstract}

Background: The aim of this study was to investigate the medium-term outcomes of laparoscopic pubocervical fascia reconstruction and sacrospinous ligament fixation with a posterior approach for severe pelvic organ prolapse (POP).

Methods: A total of 129 patients with severe POP quantitation stage III-IV who underwent laparoscopic pubocervical fascia reconstruction and sacrospinous ligament fixation with a posterior approach at Sir Run Run Shaw Hospital College of Medicine, Zhejiang University, Hangzhou, China between September 2014 and December 2018 were enrolled in this study. One hundred twenty-four patients were followed up for at least 1 year. The results and complications were recorded. Data were retrospectively reviewed.

Results: All the patients underwent laparoscopic pubocervical fascia reconstruction and sacrospinous ligament fixation with a posterior approach. The average follow-up time was $32.27 \pm 12.90$ months. The objective cure rate for those who underwent hysterectomy was $91.7 \%$ (100/109), and no patient experienced apical vaginal prolapse. The objective cure rate for those who retained uterus was $66.67 \%$ (10/15). Suture-related complications were recorded.

Conclusions: Laparoscopic pubocervical fascia reconstruction and sacrospinous ligament fixation with the posterior approach is a safe, minimally invasive, and effective method for patients with severe POP. Long-term follow-up is required to confirm the clinical effects.

\section{Background}

Pelvic organ prolapse (POP) is a common gynecological disease in middle-aged and older women, especially common among women over 50 years old of age. The main symptoms are organ prolapse in the pelvic cavity, including the uterus, bladder, and rectal prolapse. POP seriously affects patients' physical health and quality of life, increasing the financial burden for patients, and becoming a major public health concern [1].

There are many treatments for $\mathrm{POP}$, including pelvic floor muscle training, pessaries, surgery, or a combination of the abovementioned. Surgery is the most effective treatment for pelvic organ prolapse. The traditional surgical method is hysterectomy; nevertheless, after hysterectomy, the vagina loses the support of ligament, and if the weakness and defect of pelvic viscera aren't repaired, they may lead to the vaginal vault prolapse that has been recorded to occur in $6-8 \%$ of cases[2]. Over recent years, with the increasing understanding of the pelvic floor anatomy, the surgical method of POP has changed from removing extruded tissues/organs to correcting symptomatic pelvic floor defects, reconstructing vaginal anatomy, and strengthening the supporting structure of pelvic floor so as to maintain normal bladder and rectum function. Total pelvic floor reconstruction using mesh can restore pelvic floor anatomy and improve patients' quality of life. However, the serious side effects caused by the use of mesh, such as mesh exposure, erosion, and dyspareunia, have attracted increasing attention precluding a routine mesh repair in sexually active women. The Food and Drug Administration (FDA) warned in 2008 and 2011 that 
mesh should be cautiously used in total pelvic floor reconstruction. Because of the increased awareness of risk and complications associated with the mesh, gynecologists are becoming more and more cautious in using polypropylene mesh, paying more and more attention to the surgical treatment of POP by using native tissues. Gynecologists began to re-focus on the application of native tissues in the treatment of POP. There are many surgical techniques to treat POP with using native tissues, among which reconstruction of pubocervical layers and sacrospinous ligament fixation become the focus of research. These techniques can reconstruct the anatomy of the vaginal axis, restore the normal vaginal function [3], and be performed as the vaginal or abdominal procedure. The success rate in using native tissues ranges from 30-97\% [4]. Transvaginal surgery is less invasive, has less intestinal interference, less pain, faster recovery, and no scar on the abdomen. Nevertheless, due to the narrow vaginal space, it is difficult to provide adequate surgical exposure. Pelvic floor reconstructive surgery can also be performed through a transabdominal approach. Transabdominal surgery provides large vaginal space, clear vision and is considered as a relatively simple procedure; however, it is also linked with great trauma, longer operation time and hospital stay, slower postoperative recovery, and potential scarring of the abdomen. Compared to transvaginal surgery, the risk of complications increases in transabdominal surgery.

At the same time, with the introduction of laparoscopic technology, the application of laparoscopic technology in POP has been gradually increasing. While laparoscopic sacrocolpopexy with mesh has been used for many years in clinical practice, few studies have a focus on the application of native tissues in the treatment of POP with laparoscopic techniques. Over the recent 4 years, 129 cases of laparoscopic pubocervical fascia reconstruction and sacrospinous ligament fixation have been performed in Sir Run Run Shaw Hospital College of Medicine, Zhejiang University, Hangzhou, China. In order to investigate the medium-term efficacy of laparoscopic pubocervical fascia reconstruction and sacrospinous ligament fixation, follow-up longer than 1 year was conducted in 124 patients after operation. The aim of this study was to evaluate the clinical efficacy and effect of laparoscopic reconstruction on the pubocervical fascia and sacrospinous ligament fixation.

\section{Methods}

\section{Patients}

This is a retrospective longitudinal study. The analysis was performed on data from 129 patients with genital prolapse patients who underwent laparoscopic pubocervical fascia reconstruction and sacrospinous ligament fixation (LSSLF) with a posterior approach at the Department of Gynecology and Obstetrics, Sir Run Run Shaw Hospital College of Medicine, Zhejiang University, Hangzhou, China between September 2014 and December 2018. Five patients were lost to follow-up, and the missed follow-up rate was $3.9 \%$. Clinical and postoperative follow-up data of 124 patients were retrospectively analyzed. Their average age was $62.70 \pm 9.48$ years. One hundred seven cases $(86.3 \%, 107 / 124)$ were menopausal, the average menopausal time was $15.03 \pm 9.29$ years, and there was no history of hormonal therapy. The average body mass index (BMI) was $23.90 \pm 2.76 \mathrm{~kg} / \mathrm{m}^{2}$; the average gravidity was 
$3.20 \pm 2.09$, the average parity was $2.09 \pm 1.20$, and the length of POP was $3.79 \pm 5.03$ years. There were 67 cases with one or more medical comorbidities, including 56 cases of hypertension, 5 cases of coronary heart disease, 16 cases of diabetes, 5 cases of chronic obstructive pulmonary disease, 2 cases of cerebral vascular disease, and 5 cases of hysteromyoma or adenomyosis. Fourteen patients had pelvic surgery history, including 5 cases of subtotal hysterectomy, 2 cases of bilateral appendectomy, and 5 cases of myomectomy. One patient had a history of anterior colporrhaphy, and one had a history of tension-free vaginal tape sling. Evaluation of clinical symptoms and urodynamic examination revealed that 29 patients had stress urinary incontinence (SUI), 27 patients had frequent micturition, 12 patients had dysuria, 1 patient had difficult defecation, and 1 patient had fecal incontinence.

Pelvic examination was performed before the operation to evaluate the stage of POP (Table 1). The pelvic organ prolapse quantification (POP-Q) was used to determine the stage of genital prolapse [5]. All 124 patients had defects of the anterior compartment and/or middle compartment. Uterine prolapse and anterior vaginal prolapse above stage III were $79.03 \%$ and $39.52 \%$ respectively, some of the patients had prolapse in two or more than two places.

\section{Pre- and post-operative preparation}

Before the operation, a transvaginal ultrasound scan, urinary system ultrasound scan, and cervical smear were performed. If patients had vaginal erosion, estriol was prescribed for 2 weeks before the operation. After surgery, we informed patients to avoid lifting weights ( $>5 \mathrm{~kg})$ and heavy physical work within 3 months and try to control chronic cough and constipation to increase abdominal pressure.

\section{Operation Technique}

Laparoscopic operations were performed by one of the experienced surgeons in our team. All patients were intubated under general anesthesia. Intravesical catheterization was performed after successful anesthesia, with the patient being placed in the dorsal lithotomy with Trendelenburg position. An indwelling Foley catheter was placed using sterile technique after the vagina was prepped. All laparoscopic operations were performed using standard endoscopic equipment. After the establishment of $\mathrm{CO}_{2}$ pneumoperitoneum was set at a pressure of $12-14 \mathrm{mmHg}$, operations were performed with a 10 $\mathrm{mm}$ intraumbilical trocar, and three $5 \mathrm{~mm}$ trocars in the lower abdomen. A uterine manipulator was introduced through the vagina at the beginning of the operation. Before repairing the pubocervical fascia and fix sacrospinous ligament, the critical step was the identification and separation of bilateral ureters from the pelvic entrance to the deep pelvic cavity so as to avoid injury of the ureter during suture and fixation. Laparoscopic hysterectomy was performed at the same time.

Laparoscopic pubocervical fascia reconstruction is usually performed first during the routine surgical procedure. The peritoneum on the retrovesical pouch was dissected, after which the bladder-vagina space was fully dissected, and the anterior vaginal wall was completely exposed to the lower edge of the pubocervical fascia defect. The incision was made at least $4 \mathrm{~cm}$ away from vaginal fornix. The key part of blunt dissection was the cleat visualization of the internal surface of the pubocervical fascia: it had the 
glossy-like appearance, and the vaginal vessels could be clearly seen through it. We used a 1-0 nonabsorbable Ethibond Polyester Sutures with an attached needle to repair the pubocervical fascia defect. The first suture was placed at the right anterior proximal position towards the distal position exceeding the lower edge of the pubocervical fascia defect. This step was then repeated from the right side to the left side. The suture needle was inserted from the vaginal fornix but did not penetrate the vaginal wall

(Figure $1 \mathrm{~A}, \mathrm{~B}$ ). The reconstruction of the pubocervical fascia was continued with 6-8 plications depending on the patient's anatomy. If the patient requested to retain the uterus, the pubic end of the pubocervical fascia defect was sutured to the anterior part of the cervix to repair the pubocervical fascia. If the uterus was excised, the pubocervical fascia defect at the pubic end and the posterior vaginal wall fascia were sutured and fixed to the vaginal stump. After the pubocervical fascia was sutured without tying, we performed LSSLF with a posterior approach. The medial space of uterosacral ligament was fully separated from the sacral end of the uterusacral ligament to one-third to one-half so that the strong ligament structure could be observed. In the process of dissection, laparoscopic forceps can obviously touch the sciatic spine. We also used a 1-0 non-absorbable Ethibond Polyester Sutures with the attached needle in LSSLF. The first stitch on each side is of essential importance. The suture needle penetrates the right sacrospinal ligament (Figure $1 \mathrm{C}$ ), thus during suturing, it is necessary to pay attention to the depth of needle insertion should, since too deep or too wide insertion could damage the surrounding blood vessels and nerves. After the first stitch was completed, the surgeon sutured along the uterosacral ligament approximately every $1 \mathrm{~cm}$ towards the vaginal wall. The opposite side was sutured using the same method. After all the sutures were completed, hysterectomy was performed, and the vaginal stump was sutured with 1-0 absorbable suture. After vaginal stump was closed, the sutures passed through the vaginal wall, which they did not penetrate. The same-side sutures of the anterior vaginal wall and uterosacral ligament were tied. If the uterus was retained, the suture passed through the attachment point of the uterosacral ligament at the cervix. When the sutures were tied, it was necessary to ensure free tension in the sutured tissue, and to avoid suture gap, so as not to cause postoperative pelvic hernia (Figure 1 D). Posterior colporrhaphy, laparoscopic cervicectomy, laparoscopic bilateral appendectomy and other additional surgeries were performed at the same time. If ureter or bladder injury was suspected during the operation, ureteroscopy or cystoscopy was performed, and a double $\mathrm{J}$ ureteral was inserted when necessary. Length of stay, postoperative length of stay, operative time, blood loss, transfusion, the highest temperature after surgery, fever (temperature $>38^{\circ} \mathrm{C}$ on two occasions), postoperative length of the anterior vaginal wall, postoperative length of the posterior vaginal wall, days of an indwelling catheter, intraoperative and postoperative complications were recorded.

After discharge, we followed up patients through regular outpatient review. In the first year after surgery, we followed up patients every three to four months with vaginal examination and questionnaire. After that, the follow up was performed once a year. Complications, a chief complaint of postoperative discomfort, and the recurrence of pelvic organ dysfunction were recorded. We took a vaginal examination to diagnose any POP after surgery using POP-Q and informed patients to come to the hospital at any time if they experienced any discomfort during the follow-up period. The objective cure was defined as POP-Q of stage 0 after surgery. Anatomic success was defined as no apical descent greater than one- 
third into the vaginal or anterior or posterior vaginal wall beyond the hymen [6]. Recurrence was defined as the presence of POP stage II or greater.

\section{Statistical Analysis}

Statistical analysis was performed with Statistical Package for Social Science (SPSS) version 21.0 for Macintosh (SPSS, Inc., Chicago, IL, USA). Quantitative variables were expressed as mean, standard deviation and range, and qualitative variables as frequency and percentages.

\section{Results}

Laparoscopic pubocervical fascia reconstruction and sacrospinous ligament fixation with a posterior approach were successfully performed in all patients. The average operative time was $153.10 \pm 39.60$ minutes. Hysterectomy was performed in 109 patients. Among 15 patients who retained uterus, seven underwent partial cervical resection. Other operations were performed at the same time, including 7 cases of posterior colporrhaphy, 98 cases of laparoscopic bilateral appendectomy, 9 cases of laparoscopic bilateral salpingectomy, 5 cases of laparoscopic cervicectomy, 1 case of laparoscopic myomectomy. The average blood loss was $43.20 \pm 29.36 \mathrm{ml}$, and there was no need for blood transfusion. Bladders of two patients were suspected to be sutured during the operation; cystoscopy showed no suture lines in the bladder. Four cases of intraoperative ureteral injury were treated with ureteroscopy, and a double $J$ ureteral catheter was inserted. The double $\mathrm{J}$ ureteral catheter was successfully removed 3 months after the operation. The average length of the anterior vaginal wall after the operation was $5.95 \pm 0.56 \mathrm{~cm}$. The average length of the posterior vaginal wall after the operation was $6.71 \pm 0.57 \mathrm{~cm}$. All patients survived the perioperative period. The highest temperature after surgery was $37.27 \pm 0.30^{\circ} \mathrm{C}$. Postoperative length of stay was $3.56 \pm 1.01$ days. The operation data are shown in Table 2 .

There were 3 cases of postoperative urinary retention, 1 case with an indwelling catheter for 2 weeks, 2 cases with an indwelling catheter for 1 month. The average duration of an indwelling catheter for the remaining patients was $2.49 \pm 1.33$ days; patients urinated smoothly after the removal of the catheter. All patients with SUI were cured. During the follow-up period, 7 patients developed urinary incontinence, the earliest case with symptoms was 5 months after operation; this patient who had urinary incontinence before the operation, underwent the tension-free vaginal tape sling one year after the operation. The remaining patients were still under observation because of mild symptoms. In 7 patients who experienced soreness of loins, symptoms were relieved without any treatment 2-3 months after the operation. Leg pain occurred in 2 patients on the first day after the operation, which was relieved using combined therapy of mecobalamin, physiotherapy, and non-steroidal anti-inflammatory drugs for 3 months after the operation. Buttock pain occurred in 2 patients in the first week after the operation, which was relieved with the therapy of non-steroidal anti-inflammatory drugs for 1 week. Constipation occurred in 9 patients, among whom 8 improved after dietary adjustment, and one patient needed to use glycerin to help defecate. Four patients experienced pelvic heaviness; symptoms could be relieved after defecation. There was no pelvic hematoma or infection after the operation. During the follow-up period, one case of suture 
exposure and granulation occurred in the stump of the vagina within two years after the operation that was accompanied with continuous drip bleeding of the vagina, all of which disappeared after removing the thread head, and did not affect the overall effect of surgical repair.

The objective cure was defined as POP-Q of stage 0 after surgery. According to POP-Q at 3 months after the operation, the success rate was $100 \%$. The average follow-up time for 124 patients was $32.27 \pm$ 12.90 months. In the hysterectomy group, the objective cure rate was $91.7 \%(100 / 109)$ according to the definition of surgical success one year after the operation, recurrence of anterior vaginal wall prolapse was recorded for 7 patients $(6.5 \%)$, the recurrence of posterior vaginal wall prolapse was recorded for 2 patients $(1.8 \%)$, no patient had apical vaginal prolapse, one patient had a re-operation three years after the first one due to severe symptoms. In the group of patients who retained the uterus, the objective cure rate was $66.67 \%$ (10/15), five patients recurred in one year after the operation, five patients had uterine

prolapse, three of the five patients also had anterior vaginal wall prolapse, no patients had posterior vaginal wall prolapse. All the five patients were postmenopausal women, among whom four were not treated due to mild symptoms, and 1 patient accepted pessary treatment.

\section{Discussion}

POP is a common disease in elderly women, whose incidence tends to increase with age. It was reported that about $30-50 \%$ of older women over 60 years of age suffered from different degrees of pelvic floor disorders [6]. Although this disease is not life-threatening, it can seriously affect the quality of life in elderly women and impose additional financial burdens on patients. Surgery is an essential method for the treatment of moderate and severe POP. There are many surgical techniques in the treatment of POP, but still no consensus on the most effective and safe surgical approach so far. Many techniques have been proposed, especially for the treatment of vaginal vault prolapse, and the diversity of these techniques illustrates the difficulty and complexity of vaginal vault suspension.

Vaginal apex fixation is considered a critical step in reconstructive pelvic surgery. Attaching the top of the vagina so as to firm up anatomical structures can improve anterior vaginal prolapse and posterior vaginal prolapse. Currently, the accepted operations used in vaginal apex fixation are sacrospinous ligament fixation (SLF), high uterosacral suspension (HUS), sacral colpopexy (SC), SLF, among which HUS relies on the use of native tissues in reconstructive pelvic surgery.

Sacrospinous ligament fixation (SSLF) is an effective surgical treatment for the middle compartment defect that has been defined as the most popular approach for the restoration of the vaginal apex. The sacrospinous ligament, which is located in the posterior pelvic cavity with a constant position, can be clearly touched through the vagina and rectum, and it is an effective point for fixation. SSLF is usually vaginally performed due to the relatively deep position of the sacrospinous ligament and the important nerves, blood vessels, and organ around it. Since vaginal SSLF may be difficult, special and often expensive instruments, such as Deschamps needle, and the Capio suturing device, are needed to complete the operation. SSLF can also be performed abdominally. Pollak's study showed that passing 
the suture through the sacrospinous ligament under direct visualization when SSLF was performed through the abdominal approach resulted in less Intra and postoperative complications [7]. SSLF through the transabdominal approach has the advantages of large space, clear field of vision, and simple procedure; however, it may lead to severe trauma after abdominal surgery, the slow postoperative recovery, and the long hospital stay. With the development of laparoscopic surgery, the anatomical structure of the pelvic floor above levator ani muscle can be clearly obtained by laparoscopy since laparoscopic approach has excellent intraoperative visualization of pelvic anatomy, decreased blood loss, shorter hospital stay and quick postoperative recovery.

LSSLF involves two approaches to be performed: an anterior approach and a posterior approach. The anterior approach is performed by dissecting the retropubic space, paravaginal space, and lateral pelvic sidewall space to reach and identify the sciatic spine. Then posterior tissues are bluntly dissected to expose the coccygeus muscle, and the vaginal stump is sutured to the sacrospinous ligament. The range of issues that need to be separated by this surgical procedure is large, and the risk of complications such as injury of ureter, bladder, and obturator nerve, increases at the same time.

Consequently, we attempted to perform posterior sacrospinous ligament fixation. Intraoperative ureteral injury is a perioperative complication in this operation; thus, it is very important to observe the ureter before opening the peritoneum and confirming the course of the ureter. Separation of the ureter can greatly reduce ureteral injury, fistula, and pelvic infection. After the ureter is clearly identified, the peritoneum is opened at the lateral side of the uterosacral ligament, bluntly dissecting the tissue of the pararectal groove and exposing the sacrospinous ligament. Nevertheless, from the perspective of the autopsy, some researchers suggest that bladder is more likely to be injured through anterior approach. At the same time, ureter, rectum, and vascular plexus are more likely to be injured through the posterior approach $[8,9]$. In general, the rectum, which can be clearly identified during the laparoscopic approach, is less likely to incur injury. If pelvic adhesion is serious and rectal separation is difficult, a rectal probe is inserted into the rectum to identify the boundary of the rectum during the operation [10]. In surgery, the first stitch of sacrospinous ligament suture is crucial, especially the depth and width of the suture. Tissue tear can easily occur with sutures that are too shallow, while nerve and blood vessels can be easily damaged if the suture is too deep. According to our experience, the suture needle is usually located in the medial half of the sacrospinous ligament so as to avoid the injury of blood vessels and nerves during the operation. When the suture is pulled up, the fixed suture can be felt and cannot be lifted.

Over recent years, numerous studies have reported on the short-term efficacy of the sacrospinous ligament fixation. The cure rates tend to range from $69-100 \%$, with a small number of apical prolapse and a relatively high proportion of anterior vaginal wall prolapse, averaging $18.3 \%(0-42 \%)[11]$. In this study, apical vaginal prolapse was not observed in patients who underwent hysterectomy simultaneously with surgery, and the recurrence rate of anterior vaginal wall prolapse was $6.5 \%$. The recurrence in the group of patients who retained the uterus was $33.33 \%$, the recurrence of uterine prolapse was $33.33 \%$, while the recurrence of anterior vaginal wall prolapse was $20 \%$. The high recurrent rate of anterior vaginal prolapse could have been caused by the changes of vaginal axial and the backward deviation of the 
vaginal apex after SSLF, which may predispose to anterior wall descent. This also suggests that the recurrence rate of anterior vaginal prolapse after sacrospinous ligament fixation should be further investigated.

Destruction of ligaments, endopelvic fascia is the direct cause of POP, and the purpose of the operation is to repair the damaged ligaments and fascia and restore its normal anatomical structure. Therefore, most of the key repairable structures of pelvic floor support are pubocervical fascia and uterosacral ligaments, which are located above levator ani muscle level, i.e., level I and II repair. A 3D digital model showed that pubocervical fascia is the most influential structure in the onset of the median cystocele [12]. Accordingly, we attempted to reconstruct the pubocervical fascia and fix it intraoperatively on the vaginal stump. Through the reconstruction of the pubocervical fascia in operation, we wanted to reduce the recurrence rate of anterior vaginal prolapse. Several studies have reported that anterior compartment support defects are largely secondary to apical support loss [13]. Anterior vaginal wall repair was recommended in higher stage prolapse. A study reported a $31 \%$ failure rate at 12 months when anterior vaginal wall repair was not performed in higher stage prolapse. In our study, we used pubocervical fascia reconstruction plication that provided better anterior support. Hugo et al reported that pubocervical fascia reconstruction, which was used in robotic-assisted laparoscopic sacrocervicopexy, was feasible and safe. It could improve anterior and apical support, minimize the use of mesh, and improve visualization during surgery [14].

The preservation of the uterus during the operation is also a matter of concern. For women of childbearing age, pelvic floor reconstruction with uterus preservation aims at an anatomical reduction, symptom relief, and fertility preservation. In this study, premenopausal women of childbearing age, combined with partial cervical resection, achieved ideal anatomical reduction without recurrence. However, in postmenopausal women with a preserved uterus, there is an increased risk of postoperative recurrence due to postmenopausal pelvic floor tissue laxity combined with uterine gravity. For postmenopausal women, there is no fertility preservation problem, and reducing the recurrence rate is the main goal of surgery. Therefore, hysterectomy can be performed at the same time during the operation to ensure a satisfactory surgical effect. Some previous studies have also reported no significant difference in the long-term outcome and surgical satisfaction of the sacrospinous ligament fixation with or without preserved uterus [15].

The medium-long term efficacy of reconstructive pelvic surgery is an important index used to evaluate the surgical effect. David-Montefiore et al. observed the medium-term and long-term surgical effects in 51 patients, with an average follow-up time of $23.8 \pm 8$ months; $94.3 \%$ of patients had no objective recurrence [16]. Chen et al. analyzed the surgical effect among 94 cases of VSSLF 1 year after the operation, where apical compartment success rate was $94.7 \%$, and recurrence of anterior/ posterior prolapse was $16 \%$ [17]. We assume that the success rate of postoperative anatomical reduction is related to the following factors: 1 . The severity of POP before the operation is recognized as one of the important factors affecting the outcomes of reconstructive pelvic surgery. POP-Q stage III or IV is a high-risk factor for postoperative POP recurrence [18]; 2 . The site of suture: during the operation, vaginal apical fixation 
should be ensured on the sacrospinous ligament, rather than on the surrounding connective tissue. If feasible, increasing the number of suture needles can make vaginal apical fixation more stable and effective. Besides, the thickness of vaginal stump tissue may also affect the result of surgery, where the suture should be as thick as possible to suture vaginal stump tissue. With the increase in the number of cases, we found that the lowest point of suture should be lower than the Aa point when pubocervical fascia reconstruction was performed, which can significantly reduce the recurrence rate of anterior vaginal prolapse after surgery. 3 . The experience of the surgeon: The biggest challenge with laparoscopic surgery is the long learning curve. Compared with opening surgery, the laparoscopic operation is significantly more difficult, especially in the initial learning stage. In our study, we found that most of the patients experienced recurrence in the first two years after the operation. With the increase in the number of cases, the improvement of surgical skills, and the increase of the surgeon's experience, the surgeon is more skilled in laparoscopic suture and knotting, which in turn significantly reduced postoperative recurrence. 4. Anterior colporrhaphy and posterior colporrhaphy: are important factors affecting the overall outcome of the surgery. In our study, we used a one-thread continuous suture on the internal side of the pubocervical fascia with nonabsorbable sutures to provide greater firm and durability. 5 . Suture materials: Currently, non-absorbable (Prolene, braided polyester sutures, Ethibond) and absorbable sutures (Vicryl and PDS) are commonly used in reconstructive pelvic surgery. Non-absorable sutures have been associated with lower rates of recurrence compared with absorbable sutures $[19,20]$.

Previous studies have shown that although the quality of life after reconstructive pelvic surgery has no absolute relationship with the stage of POP, the subjective cure rate of POP is highly correlated with the objective cure rate. The sensation of tissue protrusion into or from the vagina, vaginal or perineal pain, pelvic pressure, pelvic heaviness, low back pain, and other mechanical symptoms are the most troublesome. These are followed by the symptoms of the lower urinary tract, including urinary retention, urinary incontinence, and similar. In this study, we restored the normal anatomical structure of the pelvic cavity, and at the same time, the symptoms of urinary incontinence and dysuria that existed in most patients before the operation was solved. The symptoms of pelvic heaviness, urination, and defecation were significantly relieved and the pelvic floor function was significantly improved. The average age of the patients in this study was $62.70 \pm 9.48$ years old, and the number of active living patients was small. Therefore, sexual function was not investigated.

The rates of adverse events in the present study are consistent with other clinical studies using native tissue in pelvic floor reconstruction [21]. Compared with the traditional sacrospinous ligament fixation, the advantages of laparoscopic sacrospinous ligament fixation with posterior approach include small incision, small dissection, good exposure, and direct vision. Injuries to the urinary systems are among the most common complications related to this surgery, including bladder injury and ureteral injury. If the injury of the urinary system is suspected during the operation, cystoscopy/ureteroscopy needs to be performed. If examination revealed that sutures penetrated the bladder and ureter obstruction, the sutures should be removed, and in some cases, the ureteral stent should be placed. In the present study, the rate of ureteral injury was $3.2 \%$. Several studies have shown that the rate of ureteral obstructions after SSLF was lower than HUS, while the rate of ureteral obstructions after HUS was 3.7-9\% [22]. Jackson [23] et al 
found that ureteral occlusion was more frequent in those patients undergoing concomitant anterior colporrhaphy. In this study, we used a concomitant pubocervical fascia reconstruction during LSSLF plicated anterior vaginal wall that may pull the ureters toward the anterior vaginal wall, which in turn shortens the distance from the vaginal stump to ureters, and increases the risk of ureteral damage. Consequently, it is very important to distinguish the ureter during the operation.

Acute neuropathic pain is also one of the common postoperative complications, especially buttock pain. Some studies have reported that the incidence of neuropathic pain was $12.4 \%$ or even higher $[20,21]$, while most patients would experience relief 4 to 6 weeks after surgery. The buttock pain after SSLF may be caused by injury to the gluteal nerve and the nerve to levator ani [24]. Nonetheless, Maria [25] found that the nearest structure to the superior margin of the midpoint of the sacrospinous ligament was the S3 nerve (median distance $3 \mathrm{~mm}$, range $0-11 \mathrm{~mm}$ ), the nearest structure to the ischial spine was the pudendal nerve (median distance $0 \mathrm{~mm}$, range 0-8 $\mathrm{mm}$ ), and the median distance from inferior gluteal nerve to the ischial spine and to the midpoint of sacrospinous ligament was $28.5 \mathrm{~mm}$ and $31.5 \mathrm{~mm}$, respectively in a cadaver study. It seems that buttock pain was caused by the injury to the branches of S3/S4. The surgeon needs to thoroughly understand the complex anatomical structure surrounding the sacrospinous ligament, limit the depth of the insertion of the needle into the ligament, and avoid extending the needle out and into the sacrospinous ligament above the upper segment, which can reduce nerve compression and postoperative acute neuropathic pain.

The impact of bowel function after surgery is also worthy of attention. Some patients tend to develop de novo constipation or de novo anal incontinence after SSLF while having no such symptoms before the surgery. In our study, the incidence of constipation after surgery was 7.3\%. David ever reported that the bowel function was unaffected after bilateral SSLF [16]. Hematoma is a serious complication in SSLF. In the present study, there was no blood transfusion and no pelvic hematoma.

There are several limitations to the present study. It was a retrospective study, so we need to be very careful in analyzing the current results and in making recommendations based on those results. Data collection from available medical records was subject to inaccuracies and incomplete data entries, which may result in incomplete data abstraction, ultimately compromising the intended data analysis. Despite the limitations of this study, we believe that reported findings provide new surgical approaches, raise awareness among physicians of the relatively common surgery-related complications, and raise awareness among physicians of the surgical approach and the relentless pursuit of the best surgical procedure. In the future, we plan to design prospective clinical studies to verify the reliability of this approach.

\section{Conclusions}

Laparoscopic pubocervical fascia reconstruction and sacrospinous ligament fixation with the posterior approach is a safe, minimally invasive, and effective method for patients with severe POP. 


\section{Abbreviations}

pelvic organ prolapse (POP)

Food and Drug Administration (FDA)

laparoscopic pubocervical fascia reconstruction and sacrospinous ligament fixation (LSSLF)

body mass index (BMI)

pelvic organ prolapse quantification (POP-Q)

sacrospinous ligament fixation (SLF)

high uterosacral suspension (HUS)

sacral colpopexy (SC)

sacrospinous ligament fixation (SSLF)

\section{Declarations}

\section{Ethics approval and consent to participate}

This study was approved by the ethical committee of Sir Run Run Shaw Hospital College of Medicine, Zhejiang University, Hangzhou, China. Individual informed consent was waived due to the retrospective study design.

\section{Consent to publish}

Not applicable

\section{Availability of data and materials}

The datasets used and analysed during the current study are available from the corresponding author on reasonable request.

\section{Competing interests}

The authors declare that they have no competing interests.

\section{Funding}

This study was financially supported by National Key Research and Development Program of China (2018YFC1004800). 
Author's Contribution

ZYS contributed to designing the study, data collection and analysis, writing of the original paper. YJH contributed to conceiving and designing the study, paper reviewing and editing. ZX, LGX, SSL, CDX and LJQ contributed to data collection. All authors read and approved the manuscript.

\section{Acknowledgements}

The authors acknowledge the help of all the co-workers.

\section{References}

1. Marchionni M, Bracco GL, Checcucci V, Carabaneanu A, Coccia EM, Mecacci F, Scarselli G: True incidence of vaginal vault prolapse. Thirteen years of experience. J Reprod Med 1999, 44(8):679-84.

2. Aigmueller T, Dungl A, Hinterholzer S, Geiss I, Riss P: An estimation of the frequency of surgery for posthysterectomy vault prolapse. Int Urogynecol J 2010, 21(3):299-302.

3. Maher C, Feiner B, Baessler K, Christmann-Schmid C, Haya N, Brown J: Surgery for women with apical vaginal prolapse. Cochrane Database Syst Rev 2016, 10(10):Cd012376.

4. Gotthart PT, Aigmueller T, Lang PF, Ralph G, Bjelic-Radisic V, Tamussino K: Reoperation for pelvic organ prolapse within 10 years of primary surgery for prolapse. Int Urogynecol J 2012, 23(9):1221-4.

5. Bump RC, Mattiasson A, Bø K, Brubaker LP, DeLancey JO, Klarskov P, Shull BL, Smith AR: The standardization of terminology of female pelvic organ prolapse and pelvic floor dysfunction. Am $\mathrm{J}$ Obstet Gynecol 1996, 175(1):10-7.

6. Nygaard I, Barber MD, Burgio KL, Kenton K, Meikle S, Schaffer J, Spino C, Whitehead WE, Wu J, Brody DJ: Prevalence of symptomatic pelvic floor disorders in US women. Jama 2008, 300(11):1311-6.

7. Pollak J, Takacs P, Medina C: Complications of three sacrospinous ligament fixation techniques. Int $\mathrm{J}$ Gynaecol Obstet 2007, 99(1):18-22.

8. De Decker A, Fergusson R, Ondruschka B, Hammer N, Zwirner J: Anatomical structures at risk using different approaches for sacrospinous ligament fixation. Clin Anat 2020, 33(4):522-9.

9. Wang Y, Wang D, Li Y, Liang Z, Xu H: Laparoscopic sacrospinous ligament fixation for uterovaginal prolapse: experience with 93 cases. Int Urogynecol J 2011, 22(1):83-9.

10. Lin LL, Phelps JY, Liu CY: Laparoscopic vaginal vault suspension using uterosacral ligaments: a review of 133 cases. J Minim Invasive Gynecol 2005, 12(3):216-20.

11. Tseng LH, Chen I, Chang SD, Lee CL: Modern role of sacrospinous ligament fixation for pelvic organ prolapse surgery--a systemic review. Taiwan J Obstet Gynecol 2013, 52(3):311-7.

12. Lamblin G, Mayeur O, Giraudet G, Jean Dit Gautier E, Chene G, Brieu M, Rubod C, Cosson M: Pathophysiological aspects of cystocele with a 3D finite elements model. Arch Gynecol Obstet 2016, 294(5):983-9. 
13. Jelovsek JE, Barber MD, Brubaker L, Norton P, Gantz M, Richter HE, Weidner A, Menefee S, Schaffer J, Pugh $\mathrm{N}$ et al: Effect of Uterosacral Ligament Suspension vs Sacrospinous Ligament Fixation With or Without Perioperative Behavioral Therapy for Pelvic Organ Vaginal Prolapse on Surgical Outcomes and Prolapse Symptoms at 5 Years in the OPTIMAL Randomized Clinical Trial. Jama 2018, 319(15):1554-65.

14. Davila HH, Abdelhameed S, Malave-Huertas D, Bigay FF, Crawford K, Friedenstab A, Lum K, Bruce L, Goodman L, Gallo T: Ultrasonography and robotic-assisted laparoscopic sacrocervicopexy with pubocervical fascia reconstruction: comparison with standard technique. J Robot Surg 2020.

15. Ng SC, Tsui KP, Huang L, Chen GD: Effects of uterine preservation on long-term subjective outcomes of sacrospinous ligament fixation for the treatment of pelvic organ prolapse. Eur J Obstet Gynecol Reprod Biol 2019, 240:167-71.

16. David-Montefiore E, Barranger E, Dubernard G, Nizard V, Antoine JM, Daraï E: Functional results and quality-of-life after bilateral sacrospinous ligament fixation for genital prolapse. Eur J Obstet Gynecol Reprod Biol 2007, 132(2):209-13.

17. Chen Y, Hua K: Medium-term outcomes of laparoscopic sacrocolpopexy or sacrohysteropexy versus vaginal sacrospinous ligament fixation for middle compartment prolapse. Int J Gynaecol Obstet 2017, 137(2):164-9.

18. Vergeldt TF, Weemhoff M, IntHout J, Kluivers KB: Risk factors for pelvic organ prolapse and its recurrence: a systematic review. Int Urogynecol J 2015, 26(11):1559-73.

19. Valecha SM, D D: A prospective observational study of sacrospinous ligament fixation. AJADD 2014, 2(4):493-502.

20. Hamdy MA, Ahmed WAS, Taha OT, Abolill ZM, Elshahat AM, Aboelroose AA: Late suture site complications of sacrospinous ligament fixation. Eur J Obstet Gynecol Reprod Biol 2019, 242:12630.

21. Barber MD, Brubaker L, Burgio KL, Richter HE, Nygaard I, Weidner AC, Menefee SA, Lukacz ES, Norton $\mathrm{P}$, Schaffer $\mathrm{J}$ et al: Comparison of 2 transvaginal surgical approaches and perioperative behavioral therapy for apical vaginal prolapse: the OPTIMAL randomized trial. Jama 2014, 311(10):1023-34.

22. Margulies RU, Rogers MA, Morgan DM: Outcomes of transvaginal uterosacral ligament suspension: systematic review and metaanalysis. Am J Obstet Gynecol 2010, 202(2):124-34.

23. Jackson E, Bilbao JA, Vera RW, Mulla ZD, Mallett VT, Montoya TI: Risk factors for ureteral occlusion during transvaginal uterosacral ligament suspension. Int Urogynecol J 2015, 26(12):1809-14.

24. Roshanravan SM, Wieslander CK, Schaffer JI, Corton MM: Neurovascular anatomy of the sacrospinous ligament region in female cadavers: Implications in sacrospinous ligament fixation. Am J Obstet Gynecol 2007, 197(6):660.e1-6.

25. Florian-Rodriguez ME, Hare A, Chin K, Phelan JN, Ripperda CM, Corton MM: Inferior gluteal and other nerves associated with sacrospinous ligament: a cadaver study. Am J Obstet Gynecol 2016, 215(5):646.e1-.e6. 


\section{Tables}

Table 1

POP-Q stages of prolapse in different parts of patients with POP

\begin{tabular}{|lllll|}
\hline & Stage I (n) & Stage II (n) & Stage III (n) & Stage IV (n) \\
\hline Uterine prolapse & 8 & 18 & 87 & 11 \\
\hline Anterior vaginal prolapse & 6 & 31 & 44 & 5 \\
\hline Posterior vaginal prolapse & 5 & 4 & 4 & 0 \\
\hline
\end{tabular}

Table 2

The data of operation

\begin{tabular}{|ll|}
\hline Average operative time & $153.10 \pm 39.60 \mathrm{~min}$ \\
\hline Average blood loss & $43.20 \pm 29.36 \mathrm{ml}$ \\
\hline Ureteral injury $(\mathrm{N})$ & 4 \\
\hline Bladder injury $(\mathrm{N})$ & 0 \\
\hline Postoperative length of stay & $3.56 \pm 1.01$ days \\
\hline Transfusion & 0 \\
\hline The highest temperature after surgery & $37.27 \pm 0.30^{\circ} \mathrm{C}$ \\
\hline Average length of the anterior vaginal wall & $5.95 \pm 0.56 \mathrm{~cm}$ \\
\hline Average length of the posterior vaginal wall & $6.71 \pm 0.57 \mathrm{~cm}$ \\
\hline
\end{tabular}

Figures 

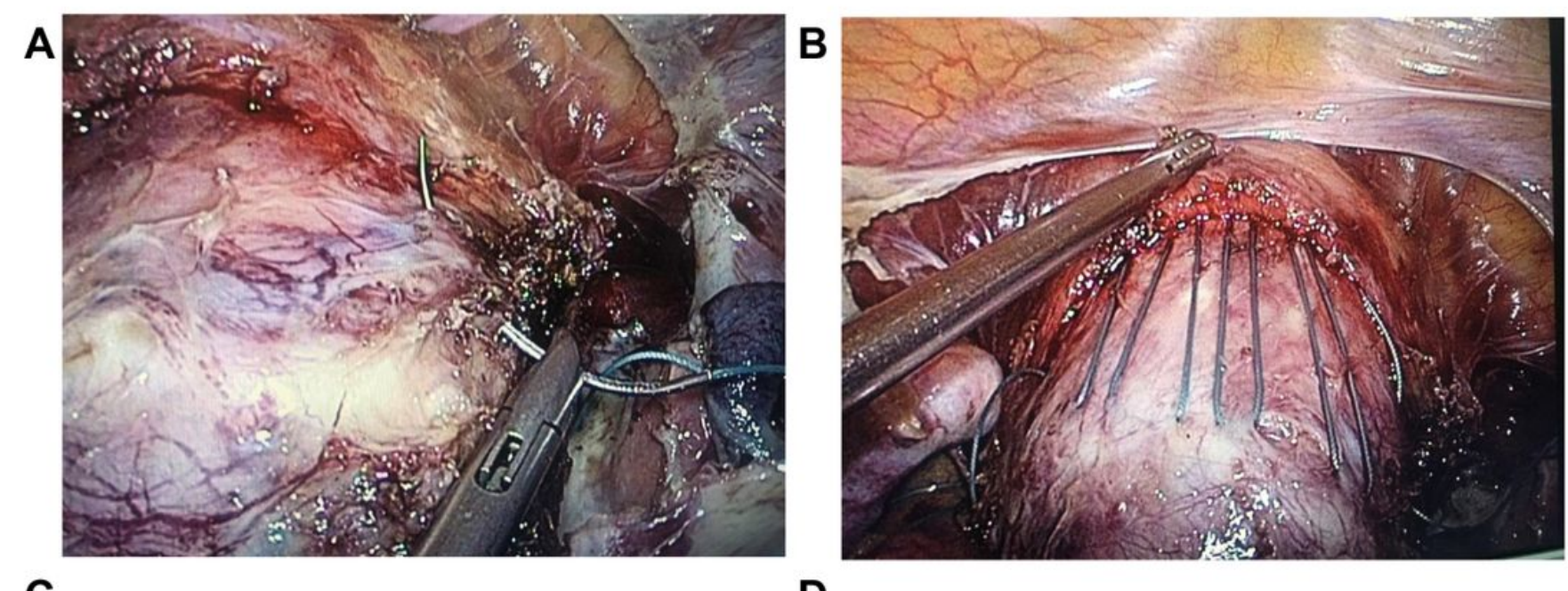

C
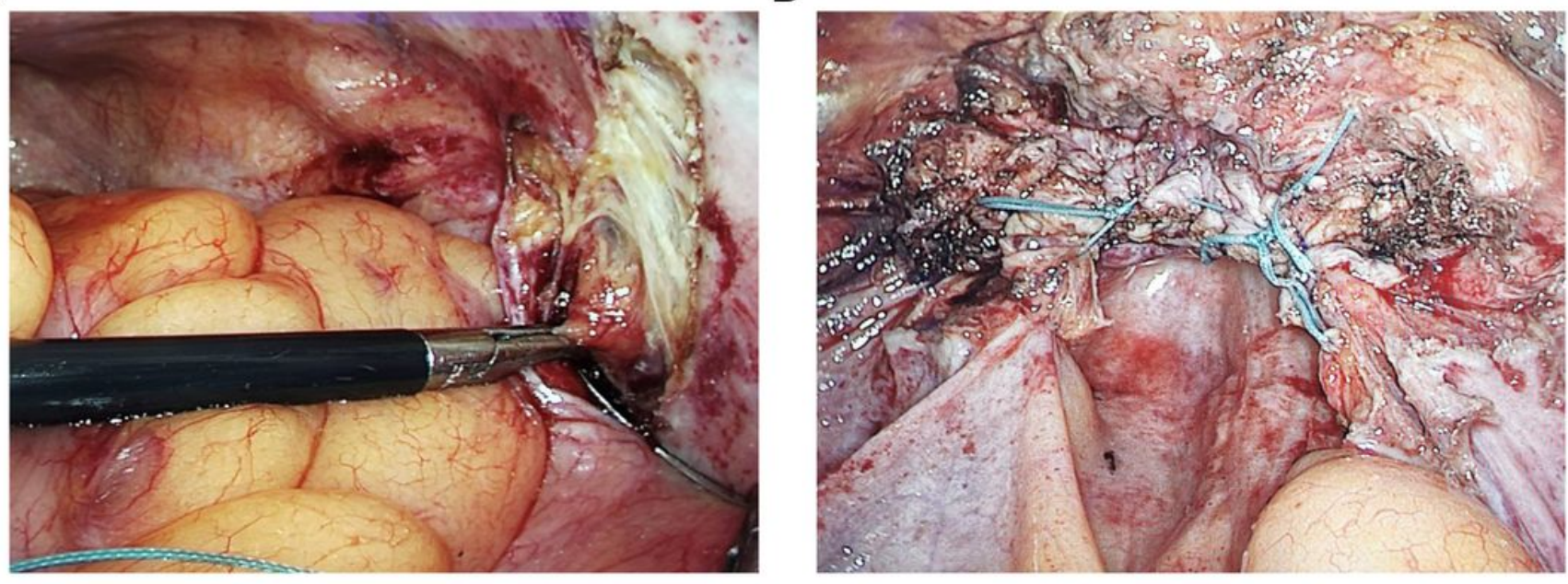

Figure 1

(A) The first suture was passed through the edge of anterior vaginal wall. (B) The reconstruction of the pubocervical fascia is continued with 6-8 plications. (C) The needle is passed through the right sacrospinous ligament with posterior approach. (D) Bilateral sacrospinous ligament fixation is completed. 